\title{
Geheimwaffe zum Erfolg
}

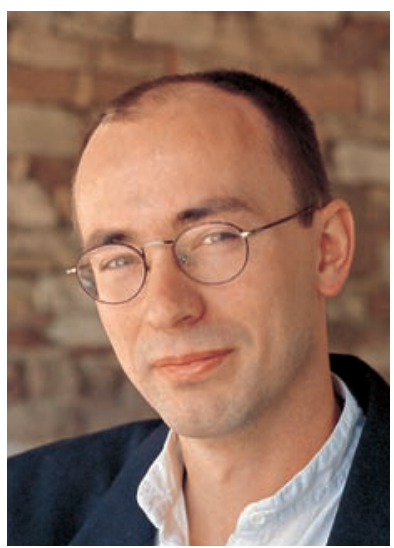

Dr. med. Dieter Schmid, Redaktionsleitung
Ist das nicht schmeichelhaft? Unser Staat traut Ihnen einiges zu! In den nächsten fünf Jahren gehen in Deutschland über 40.000 Ärzte in den Ruhestand. Die Arbeit, die deswegen liegen bleibt, soll durch die nachrückende Generation erledigt werden. Wie das gehen soll, wissen die Verantwortlichen allein. Schon jetzt können an unseren Kliniken tausende Planstellen nicht besetzt werden. Die Folgen für die verbleibenden Ärzte sind mehr Dienste und längere Arbeitszeiten. Die gute Nachricht: Mittelfristig wird der Leidensdruck auf die Kliniken so groß werden, dass ihnen gar nichts anderes mehr übrig bleibt, als die Arbeitsbedingungen zu verbessern. In unserem Artikel „Keiner da?“ auf S. $\mathbf{3 0}$ beschreiben wir, welche Maßnahmen Arbeitgeber ergreifen, um für Sie attraktive Stellen zu schaffen.

Einer der größten Arbeitgeber für Ärzte hierzulande ist die Deutsche Bundeswehr. Als Sanitätsoffizier hat man eine breite Palette von Aufgaben, die Bezahlung stimmt und auch die Arbeitszeiten sind in Ordnung. Trotzdem schrecken viele vor dem Gedanken zurück, als Arzt eine Uniform zu tragen. Die ausgeprägte Phobie gegen alles Militärische bei den meisten Medizinern ist dafür sicher ein wichtiger Grund. Zudem ist es eben nicht jedermanns Sache, regelmäßig in Feldlagern im Ausland zu arbeiten, wo man tagsüber mit der Pistole am Gürtel Patienten versorgt und nachts wegen Raketenangriffen in Bunkern unterschlüpfen muss. Damit Sie sich selbst ein ausgewogenes Bild machen können, stellen wir Ihnen die „Weißkittel in Tarnfarben“ auf S. 24 ausführlich vor.

Was dem Soldaten sein Kampfverband, ist vielen Studenten ihre Lerngruppe. Manche Prüfungsaspiranten sehen in diesen Zusammenkünften eine Geheimwaffe gegen die Examina dieser Welt. In unserer aktuellen Online-Umfrage möchten wir die Vor- und Nachteile dieser Lernform diskutieren (siehe unten). LernPädagoge Dr. Stangl gibt im Artikel „Kaffeeklatsch oder Powertruppe“ Tipps, wie man solche Treffen erfolgreich gestaltet (S. 10). Zum Beispiel: Wenn man merkt, dass man mit der Lernerei nicht mehr weiterkommt, solle man Schluss machen und zusammen ein Bier trinken gehen. Das können sicher auch Lerngruppenverächter unterschreiben ...

In diesem Sinne wünsche ich Ihnen ein ebenso erfolgreiches wie angenehmes Sommersemester 2008!

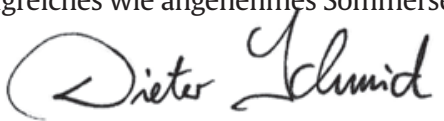

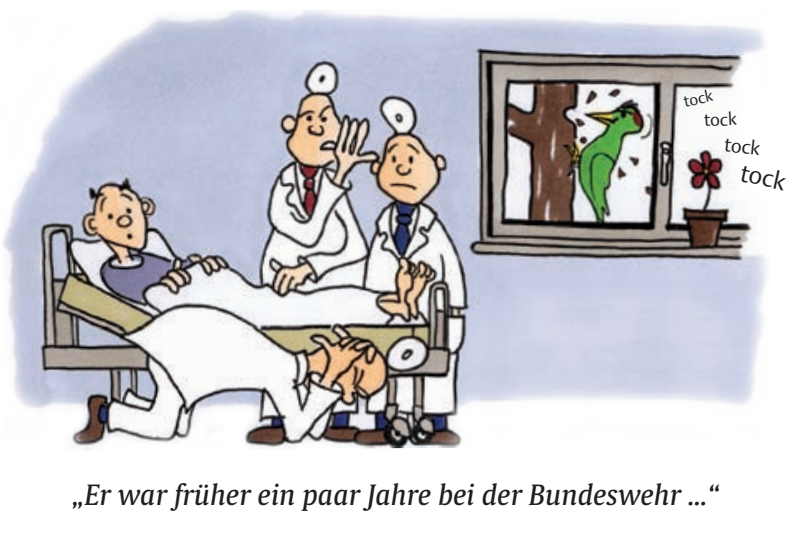

ONLINE-UMFRAGE

\section{Was bringen Lerngruppen?}

Für viele Studenten ist ihre Lerngruppe eine eingeschworene Gemeinschaft, ohne die sie die Tiefpunkte auf dem Weg zu den diversen Examina niemals überwinden würden. Andere meinen, dass die Gruppe ihnen das Genick gebrochen hat. Am heimischen Schreibtisch hätten sie sich die Fakten fürs Physikum wesentlich schneller und kompakter „reinpfeifen“ können. Wie stehen Sie zum Lernen im Team? Sind Sie überzeugter Lerngruppenlerner? Oder sagen Sie: Lerngruppe - nie wieder! f Glauben Sie, dass man sich deutlich erfolgreicher auf eine Prüfung vorbereiten kann, wenn man regelmäßig in einer Lerngruppe lernt?

Stimmen Sie ab und sagen Sie Ihre Meinung unter: www.thieme.de/viamedici/aktuelles/aktion/ miniumfrage2_08.html

\section{$f$ Ergebnis der Umfrage in Via medici 1.08:} Unsere Frage war: Soll die Anzahl der PJ-Tertiale im Ausland beschränkt werden? Über 60 \% der UmfrageTeilnehmer stimmten dagegen, $36 \%$ befürworten diese Regelung. Die Beschränkungsgegner argumentierten, dass im Ausland die Ausbildung häufig besser sei. Ein Student: „Im Chirurgie-Tertial in Kapstadt habe ich an zwei Tagen mehr gelernt als viele PJler an der Uni in einem Tertial." Die Befürworter halten dagegen, dass im PJ ein gewisser Standard gewahrt werden müsse und zudem ein Inland-Tertial Sprungbrett für eine gute Stelle sein könne. Ein Fan des Inland-PJs moniert, dass PJler, die Indien „bereist“ hätten, angesehener seien als die, die hier die Stellung halten. Alle Kommentare unter: .../aktuelles/aktion/miniumfrage1_08.html 